\title{
In-line Mach-Zehnder Interferometer based on Hybrid Structures for Refractive Index Sensing
}

\author{
A.H.M. Nasir, M.R. Mokhtar, N. S. M. Rodzi
}

\begin{abstract}
A Mach-Zehnder interferometer (MZI) built using several concatenated different structures is proposed as a refractive index sensor. This sensor is comprised of a microbubble, a section of reduced cladding fiber (RCF) and a core-offset single-mode fiber (SMF). These structures are joined together through specialized arc fusion splicing procedures. The sensor is characterised by immersing it in Cargille oil with refractive index values ranging from 1.30 to 1.39 . The sensor exhibits linearity in respect to the refractive index changes, with a good sensitivity of $144.42 \mathrm{~nm} / \mathrm{RIU}$. The proposed MZI has the advantages of cost effective, repeatable fabrication, compact size and high sensitivity, which make it a promising sensor.
\end{abstract}

Index Terms: Hybrid optical sensor, Mach-Zehnder interferometer, Microbubble, Reduced cladding fiber, Refractive index sensor

\section{INTRODUCTION}

The optical sensing technology has been utilized in various practical applications throughout the years. Furthermore, the fiber optic sensor is one of the well-known sensing technologies that have undergone vast development with many improvement features to increase the sensitivity of the sensor. A refractive index (RI) sensor is a device utilized to detect the change in RI of the ambient environment. There are several schemes of Mach-Zehnder interferometer (MZI)-based RI sensor that have been developed such as concatenated long period gratings (LPG) and fiber Bragg gratings (FBG) [1], FBG spliced with two core-offset structures [2], a down taper between two microbubble structures [3], standard single-mode fiber (SMF) sandwiched between two multi-mode fibers (MMFs) [4], tapered photonic crystal fiber (PCF) in between two sections of SMF [5], and microbubble-MMF-microbubble [6]. The MZI sensor invites interests as it has a good sensitivity, corrosion resistance, compact size, low-cost, easy fabrication, and immune to electromagnetic interference. The MZI is also a multipurpose sensor as it can be used in sensing various parameters such as RI sensor [6]-[8], temperature sensor [9], curvature sensor [10] and pressure sensor [11]. These sensors are introduced in order to replace the conventional electrical refractive index sensor due to their various significant advantages.

Revised Manuscript Received on July 29, 2019.

A.H.M. Nasir, Faculty of Engineering, Multimedia University, Cyberjaya, Malaysia.

M.R. Mokhtar, Research Institute for Digital Connectivity, Multimedia University, Cyberjaya, Malaysia.

N. S. M. Rodzi, Faculty of Engineering, Multimedia University, Cyberjaya, Malaysia.
This paper presents a development and investigation of a new structure of a fiber optic sensor which is uniquely fabricated by concatenating different fiber structures to form a MZI The structures formation is realized through specific arc fusion splicing procedures to form a microbubble, a section of reduced cladding fiber ( $\mathrm{RCF}$ ), and a core-offset SMF. The performance of the proposed fiber optic sensor is assessed in terms of its sensitivity and linearity in a liquid refractive index sensing experiment.

\section{DEVICE PRINCIPLE AND FABRICATION}

\section{A. Working Principle}

An in-line MZI configuration requires coupling of light between the core and cladding modes. The schematic diagram of the proposed optical sensor is illustrated in Fig. 1. The mechanism to provide the coupling can be achieved through a microbubble. The microbubble has a disrupted waveguide structure in a form of an up-tapered diameter section that causes some portion of propagating light to be diverted into the cladding. The cladding modes are excited when light is coupled into the cladding of the fiber. Henceforth, the light in the core and cladding modes travel along the RCF. This RCF region provide a sensing region with enhanced sensitivity due to expanded evanescent field for interaction with the surrounding medium. Subsequently, some of the light from cladding mode will be re-coupled back at the core-offset interface for interference to take place Finally, the accumulated phase difference between the core and cladding modes at the core-offset point produces an interference spectrum with multiple dips. The phase difference can be described as,

$$
\varphi^{j}=\frac{2 \pi\left(n_{c o r e}^{e f f}-n_{c l, j}^{e f f}\right) L}{\lambda}
$$

where $n_{c o r e}^{\text {eff }}$ and $n_{c l, j}^{\text {eff }}$ are the effective refractive index of the core and the $j$-th cladding modes respectively, $\lambda$ is the wavelength of the light source and $L$ is the interferometer length. 


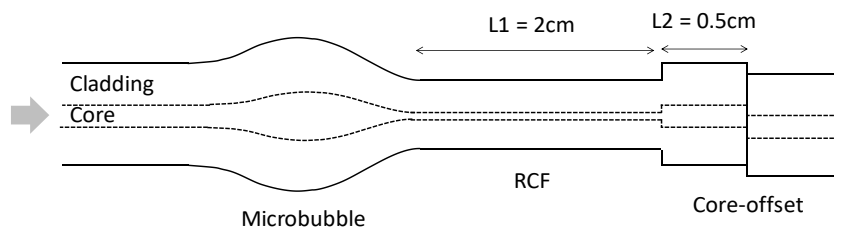

Fig. 1. Schematic diagram of the proposed optical sensor.

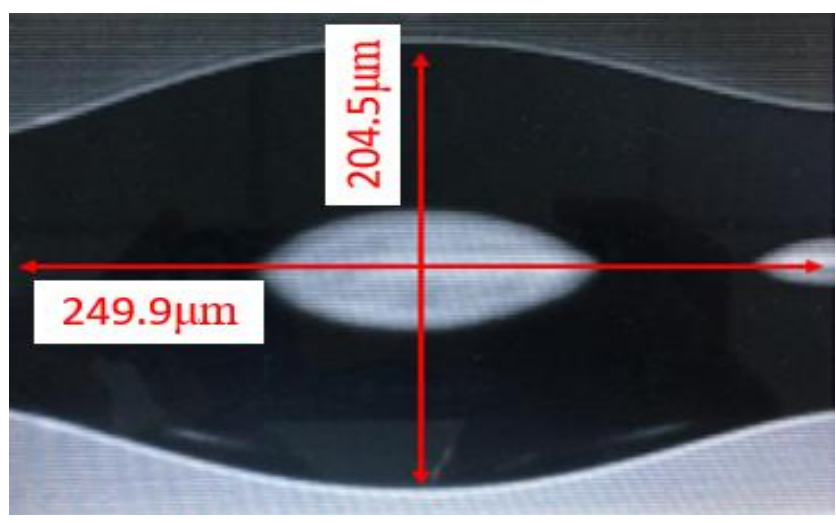

Fig. 2. The microbubble between SMF and RCF produced by using pre-arc discharge approach.

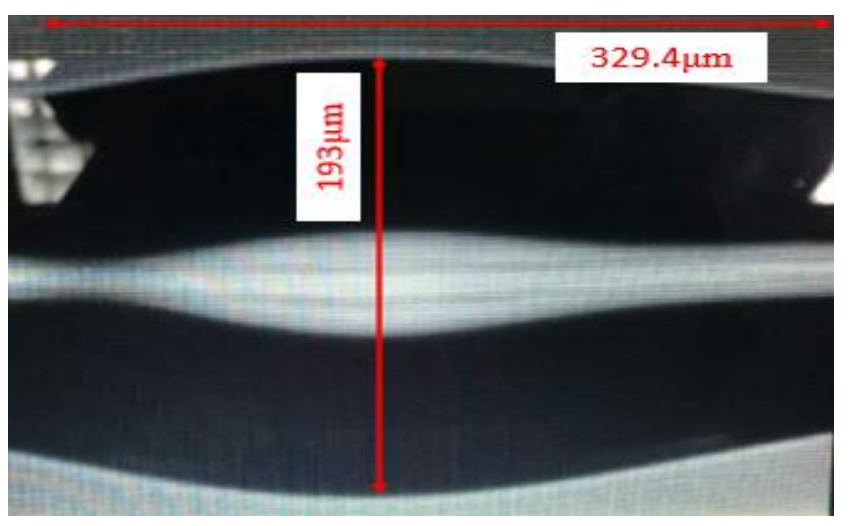

Fig. 3. The microbubble between SMF and RCF produced by using multiple arc discharge approach.

\section{B. Fabrication of the Sensor}

The MZI is fabricated by simply using a fusion splicer, whereby different splicing points along the sensor require their own unique splicing procedure. For the first mode coupling point, namely the microbubble, there are two possible approaches for fabricating this structure in between SMF and RCF, which are the pre-arc approach, and the multiple arc approach. For both of these approaches, a standard SMF with core/cladding diameter of $8 / 125 \mu \mathrm{m}$ and a RCF with core/cladding diameter of $5 / 86 \mu \mathrm{m}$ are used. The fusion splicer being used is the Sumitomo Type-36 operated in manual mode. In detail, to produce the microbubble via pre-arc method, firstly a standard SMF is placed at one side of the splicer. An arc power of 64 steps is applied to the fiber to form a spherical structure. Then, the SMF is then removed and replaced with a RCF. Likewise, an arc is applied to it with the arc power of 10 steps. Finally, both of these inchoate fibers are placed onto the splicer and spliced together to form the desired microbubble structure. The parameters for the final splicing are as follow: arc power equals to 11 steps, fusion duration of $6 \mathrm{~s}$ and overlap of $80 \mu \mathrm{m}$. The structure fabricated using pre-arc discharge approach is shown in Fig. 2. Another approach which is the multiple arc method requires a totally different procedure. In this approach, both SMF and RCF are placed onto the splicer. These fibers are spliced together with an arc power

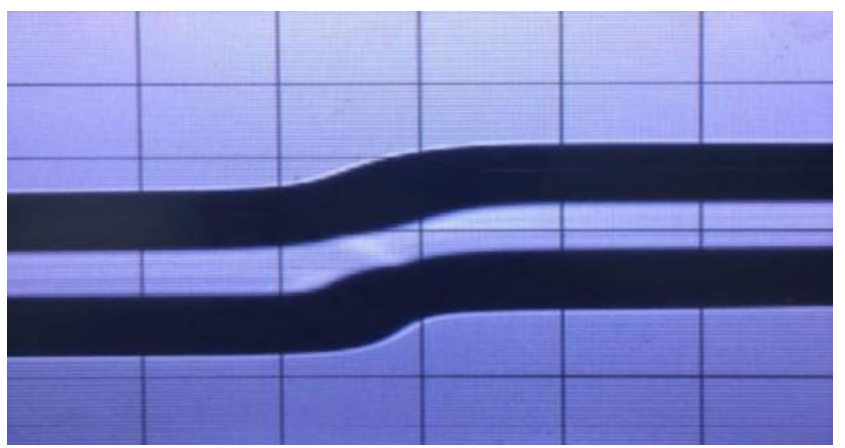

Fig. 4. The core-offset structure.

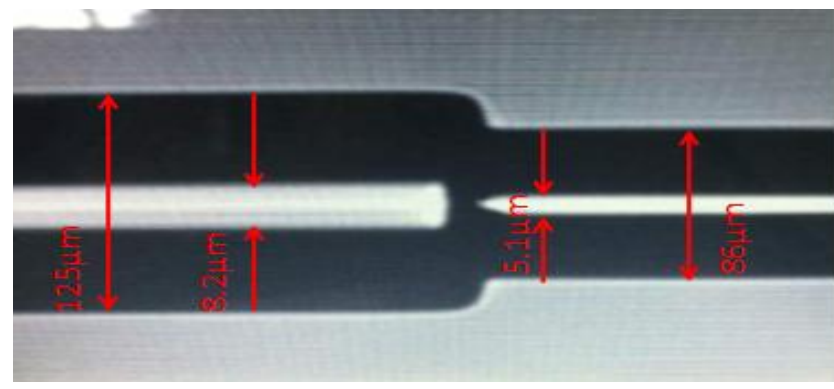

Fig. 5. The SMF-RCF splicing joint.

equals to 11 steps, fusion duration of $2.50 \mathrm{~s}$ and overlap of 80 $\mu \mathrm{m}$. The arc is applied 10 times in order to achieve the desired dimension of the microbubble. The structure fabricated using the multiple arc discharge approach is shown in Fig. 3. It is important to note that the microbubble by multiple arc discharge is chosen for its better coupling efficiency.

Finally, for the power combining point, the core-offset structure is fabricated by using the fusion splicer of model Sumitomo Type 81-C. The core-offset procedure uses the similar default parameters to splice two standard SMFs. The only significant difference is the introduction of a lateral offset. Referring to the work of Lili Mao [13], the offset should be arranged at the y-axis, while the fibers should be aligned at the x-axis. One of the SMF was displaced down $40.69 \mu \mathrm{m}$. The fabricated core-offset structure is shown in Fig. 4. This core-offset structure is connected with the RCF by setting the splicer with the arc power of 11 steps, fusion duration of $0.40 \mathrm{~s}$, pre-fusion duration equals to $1.0 \mathrm{~s}$ and overlap of $80 \mu \mathrm{m}$, which produce a joint as shown in Fig. 5. The length, L1 between the microbubble structure and the RCF-SMF joint, and the length, L2 between the RCF-SMF joint and the core-offset structure are chosen based on an experiment wherein these lengths are varied and the corresponding transmission spectra are observed. The length

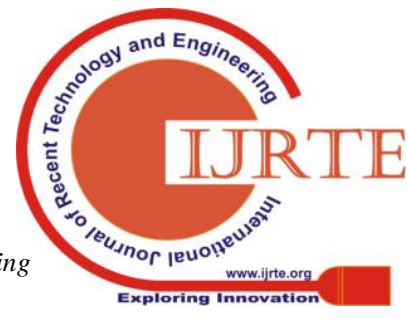


should be as short as possible to increase the compactness of the sensor which is one of the design goals. For this purpose, four sensors are prepared with the following lengths: 1) $\mathrm{L} 1=1 \mathrm{~cm}, \mathrm{~L} 2=0.5 \mathrm{~cm}, 2) \mathrm{L} 1=1 \mathrm{~cm}, \mathrm{~L} 2=1 \mathrm{~cm}, 3) \mathrm{L} 1=2 \mathrm{~cm}$, $\mathrm{L} 2=0.5 \mathrm{~cm}, 4) \mathrm{L} 1=2 \mathrm{~cm}, \mathrm{~L} 2=2 \mathrm{~cm}$. The developed MZI sensors are characterized by using a tunable laser source (Yenista

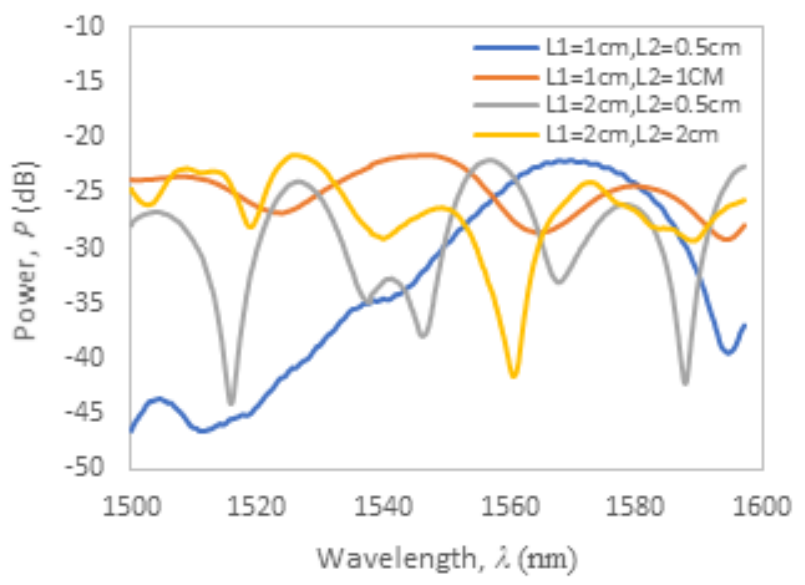

Fig. 6. The transmission spectra of the sensors in correspond to different dimensions.

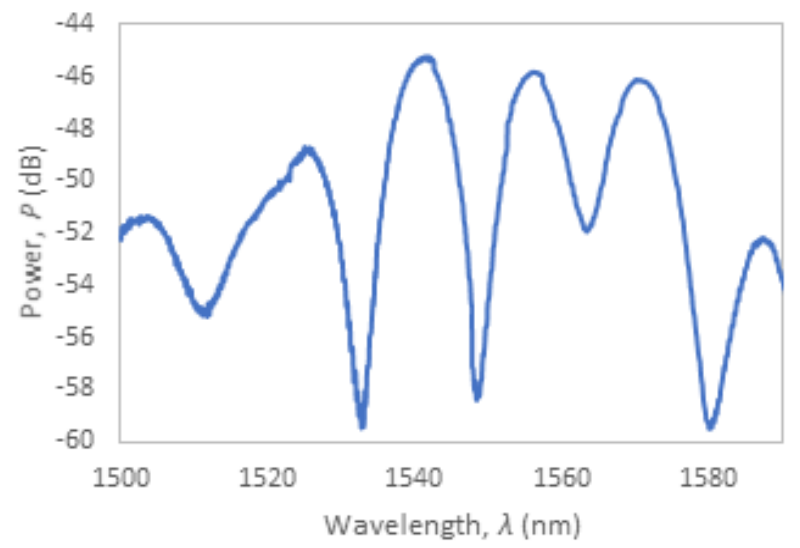

Fig. 7. The transmission spectrum of the sensor prototype.

Optics-Tunics T100S-HP). The observed wavelength window is from $1500 \mathrm{~nm}$ to $1600 \mathrm{~nm}$. An optical component tester (Yenista Optics-CT400) is used to sweep the wavelength and the resolution is set to $128 \mathrm{pm}$. The results are shown in Fig. 6, which shows that the optimum lengths are $\mathrm{L} 1=2 \mathrm{~cm}$ and $\mathrm{L} 2=0.5 \mathrm{~cm}$ since the spectrum shows a good interference dips visibility and a fair total length.

The number of interference dips will increase in number when the length of the structure is longer [6]. This theory is proven in this experiment. The two sensors with $\mathrm{L} 1=2 \mathrm{~cm}$ have more interference dips. Furthermore, the free spectral range of spectrum will decrease as the length of the sensor increases [12]. The claim is supported in this experiment since the free spectral range (FSR) is smaller for the sensors with longer length. The FSR can be described as,

$$
\Delta \lambda=\frac{\lambda^{2}}{\Delta n_{j}^{e f f} L}
$$

As can be seen from Fig. 7, the chosen MZI design produces transmission spectrum with five interference dips within the scanning window. The dips are at wavelength $1511.742 \mathrm{~nm}, 1532.731 \mathrm{~nm}, 1548.438 \mathrm{~nm}, 1563.366 \mathrm{~nm}$ and $1580.019 \mathrm{~nm}$. These dips are the result of accumulated phase difference along the interaction/sensing length.

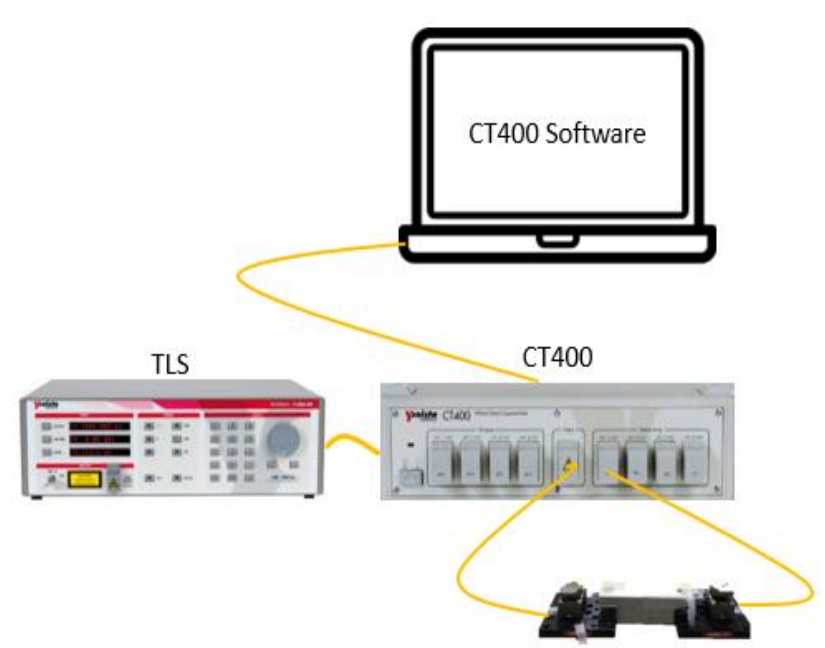

Fig. 8. The experimental set-up for refractive index sensing experiment.

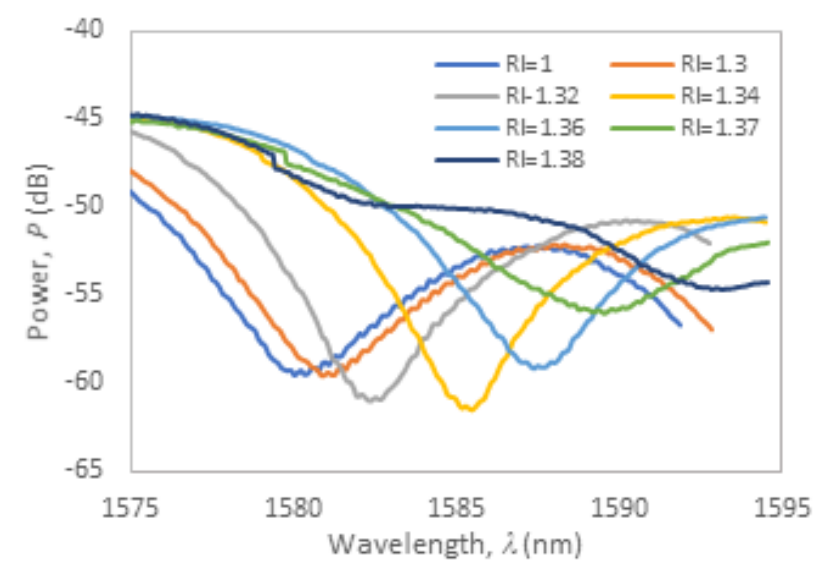

Fig. 9. The chosen wavelength window for refractive index sensing, showing the red shift of the interference dip.

\section{SENSING EXPERIMENT AND RESULTS}

In order to sweep the TLS in the range of $1500 \mathrm{~nm}$ to 1600 $\mathrm{nm}$, the optical component tester is used, which is a control instrument that is capable of sweeping continuously from $1240 \mathrm{~nm}$ to $1680 \mathrm{~nm}$ by connecting it to TLS. This device is vital for the measurement to be carried out within the desired band. The optical component tester is connected to a laptop via a GPIB cable. The driver software used to record the spectrum is installed in the laptop. Both ends of the fiber under test are connected to the output port (APC) and one of the detector array ports (PC) of the optical component tester. 
This setup is illustrated in Fig. 8.

The in-line MZI sensor was immersed in different values of Cargille RI liquids. The RI values are 1, 1.3, 1.32, 1.34, $1.36,1.37$, and 1.38. Inherently, a stage was set-up in order to maintain the sensor in a fixed position. The sensor was placed onto a clean microscope sample glass plate. Prior to that, the sensor was cleaned using a lint-free tissue with alcohol. The sensor was securely held by using two fiber holders.

Then,

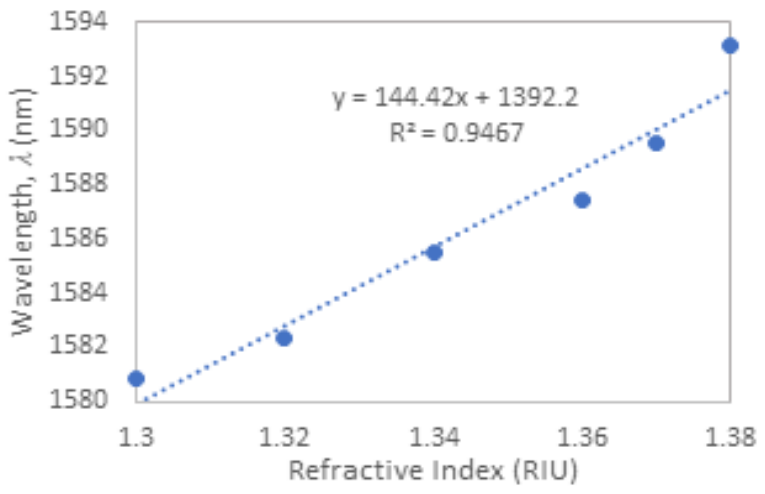

Fig. 10. The relationship between wavelength shift of the interference dip and refractive index.

both ends of the sensing region were further fixed by using scotch tape.

The MZI structure relies on multimode interference, which indicates that the multiple dips have different sensitivities [2]. By the same token, in correspond to Fig. 7, the interference dip at $1580.019 \mathrm{~nm}$ was chosen to analyze the change in refractive index since it has the best visibility of wavelength shifts. The chosen interference dip has a transmission loss of $13.38 \mathrm{~dB}$. During the course of the experiment, the room temperature was kept constant at $20^{\circ} \mathrm{C}$. The experiment started with refractive index of 1 (air) followed by the subsequent RIs. Fig. 9 shows the interference dip shifts towards the longer wavelength region as the refractive index value increases.

The sensor shows a linear relationship between the refractive index and the wavelength of the interference dip that is red-shifted. Based on the linear graph shown in Fig. 10 , the sensitivity of the MZI sensor is $144.42 \mathrm{~nm} / \mathrm{RIU}$ which is relatively high and the coefficient of determination, $\mathrm{R}^{2}$ is 0.9467 . The sensitivity is given by the slope of linear plotting of the wavelength of the interference dip against the variation of refractive index. This strengthen the theory that the sandwiched RCF in the middle of the sensor is able to extend the evanescent field. This is due to the RCF diameter that is a lot smaller than the microbubble and SMF. The RCF fiber can be said to act like a down taper. According to L. Li et al. [14], a taper that has smaller diameter to standard SMF may cause the change in distribution of radial RI and form an extended evanescent field. The light in the cladding of the $\mathrm{RCF}$ and the evanescent wave are now closer to the outer field since the cladding diameter is a lot smaller. The different RI liquids being applied at the surface of the RCF achieve more interaction with the extended evanescent field, which in turn affects the light in the cladding mode more greatly. Therefore, this effect will increase the overall sensitivity of the sensor.

\section{Conclusion}

An RI sensor based on in-line MZI has been successfully developed by concatenating multiple different types of fiber optic structures by disparate fusion splicing methods. The spliced structures are microbubble, a section of RCF, and a core-offset SMF. The spectral response analysis of the RI measurement experiment with values ranging from 1.3 to 1.38 shows red shift of the interference dip wavelength, which exhibits a high sensitivity of $144.42 \mathrm{~nm} / \mathrm{RIU}$ and excellent linearity. The proposed sensor is also potentially low cost and has good repeatability.

\section{ACKNOWLEDGMENT}

The authors would like to express the gratitude to the members of the Fiber Optic Research Center in Multimedia University (MMU). The authors are grateful for the use of equipment supported by the Ministry of Higher Education (MOHE), Malaysia, under Fundamental Research Grant Scheme [FRGS/2/2014/TK03/MMU/01/1].

\section{REFERENCES}

1. Fu, M., "Refractive Index Sensing Based on the Reflectivity of the Backward Cladding-Core Mode Coupling in a Concatenated Fiber Bragg Grating and a Long Period Grating," IEEE Sensors Journal, vol. 12, no. 5, 2012, pp. 1415-1420.

2. Yao, Q., Meng, H., Wang, W., Xue, H., Xiong, R., Huang, B., Tan. C. Huang, X., "Simultaneous measurement of refractive index and temperature based on a core-offset Mach-Zehnder interferometer combined with a fiber Bragg grating," Sensors and Actuators A: Physical, vol. 209, 2014, pp. 73-77.

3. S. Gao, W. Zhang, P. Geng, X. Xue, H. Zhang and Z. Bai, "Highly Sensitive In-Fiber Refractive Index Sensor Based on Down-Bitaper Seeded Up-Bitaper Pair," IEEE Photonics Technology Letters, vol. 24 no. 20, 2012, pp. 1878-1881.

4. Villatoro, J., \&Monzón-Hernández, D., "Low-cost optical fiber refractive-index sensor based on core diameter mismatch," Journal of Lightwave Technology, vol. 24, no. 3, 2006, pp. 1409.

5. Wang, Q., Kong, L., Dang, Y., Xia, F., Zhang, Y., Zhao, Y., Hu, H., Li, J., "High sensitivity refractive index sensor based on splicing points tapered SMF-PCF-SMF structure Mach-Zehnder mode interferometer," Sensors and Actuators B: Chemical, vol. 225, 2016, pp. 213-220.

6. Y. Yorozu, Shao, M., Qiao, X., Fu, H., Liu, Y., Zhao, X., \& Yao, N., "High sensitivity refractive index sensing of Mach-Zehnder interferometer based on multimode fiber core sandwiched between two waist-enlarged fiber tapers," Optics Communications, vol. 311, 2013, pp. 359-363.

7. P. Wang, G. Brambilla, M. Ding, Y. Semenova, Q. Wu, and G. Farrell, "Investigation of single-mode - multimode - single-mode fiber structures and their application for refractive index sensing," J. Opt. Soc. Am. B, vol. 28, no. 5, 2011, pp. 1180-1186.

8. Y. Zhao, L. Cai, and H. F. Hu, "Fiber-Optic Refractive Index Sensor Based on Multi-Tapered SMS Fiber Structure," IEEE Sens. J., vol. 15, no. 11, 2015, pp. 6348-6353

9. S. Zhu, F. Pang, and T. Wang, "Single-mode tapered optical fiber for temperature sensor based on multimode interference," in Proc. SPIE Optical Sensors and Biophotonics III, vol. 8311, 2011, pp. 83112B.

10. R. Wang et al., "Highly Sensitive Curvature Sensor Using an In-Fiber Mach-Zehnder Interferometer," IEEE Sens. J., vol. 13, no. 5, 2013, pp. 1766-1770

11. Z. Li et al., "Highly-sensitive gas pressure sensor using twin-core fiber

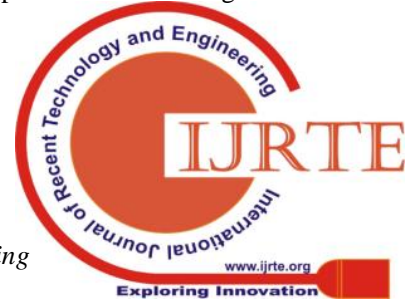


based in-line Mach-Zehnder interferometer," Opt. Express, vol. 23, no. 5, 2015 , pp. 6673.

12. Kang, Z., Wen, X., Li, C., Sun, J., Wang, J., \& Jian, S., "Up-taper-based Mach-Zehnder interferometer for temperature and strain simultaneous measurement," Applied Optics, vol. 53, no. 12, 2014, pp. 2691.

13. Mao, L., Lu, P., Lao, Z., Liu, D., \& Zhang, J., "Highly sensitive curvature sensor based on single-mode fiber using core-offset splicing," Optics \& Laser Technology, vol. 57, 2014, pp. 39-43.

14. Li, L., and Sun, X., "Investigation on the Tapered Fiber Evanescent-Field Sensor Based on the Comsol Software," in Proc. Symposium on Photonics and Optoelectronics, Shanghai, 2012, pp. 1-3.

\section{AUTHORS PROFILE}

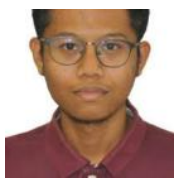

A.H.M. Nasir received the B. Eng. Degree (First Clas Honors) from the Faculty of Engineering, Multimedia University, Malaysia in 2018.

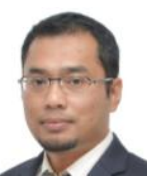

M. R. Mokhtar received the B. Eng. Degree (First Class Honors) from the Department of Electronic Engineering, King's College London, U.K., in 1998 and the Ph.D. degree in Optoelectronics from University of Southampton, U.K., in 2005. He became a Research Fellow at City University London, U.K. for a year in 2009. He is currently a Professor at the Research Institute for Digital Connectivity, Multimedia University, Malaysia. His main research areas include optical sensors and optical communications.

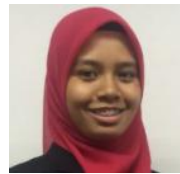

N. S. M. Rodzi received the B. Eng. Degree (Honors) from the Faculty of Electrical Engineering, Universiti Teknologi Mara, Malaysia in 2016. She is currently pursuing a Master's degree at the Faculty of Engineering, Multimedia University, Malaysia. Her main research areas include optical sensors and optical communications 